\title{
Ubiquitous Support for Midwives to Leverage Daily Activities
}

\author{
Abdullah Al Mahmud \\ ID-StudioLab \\ Industrial Design Engineering \\ Delft University of Technology \\ 2628 CE Delft, the Netherlands \\ a.almahmud@tudelft.nl

\section{David V. Keyson} \\ ID-StudioLab \\ Industrial Design Engineering \\ Delft University of Technology \\ 2628 CE Delft, the Netherlands \\ d.keyson@tudelft.nl
}

\begin{abstract}
In this paper we present preliminary outcomes concerning the design of a support system for midwives in the Netherlands to carry out daily activities. The purpose of our design is to make the workflow of midwives more efficient. Our user studies confirm that the current workflows of midwives to support pregnant women lack efficiency. The most obvious barrier is the lack of an integrated IT system to provide daily care to pregnant women. Based on the findings we propose some solutions that may help midwives to perform their daily tasks more efficiently.
\end{abstract}

\section{Keywords}

Midwives, workflow, pregnancy, prenatal care

\section{ACM Classification Keywords}

H5.2. [Information Interfaces and Presentation]: User Interfaces.

\section{Introduction}

A key aspect to improve maternal health is to make use of the role of skilled health professionals, specifically midwives. Women around the world depend on the expertise of midwives for both prenatal and neonatal care. In the Netherlands, midwives are independent medical practitioners who work either in a midwifery practice centre or in a hospital. Approximately $78 \%$ of 
pregnant women start their maternity care with a primary care setting where $44 \%$ start labour in primary care, and finally $33 \%$ of women give birth under supervision of a primary care midwife [1]. Midwives are trained to provide maternity care to women whose pregnancy and childbirth are uncomplicated including antenatal, intrapartum and postnatal care to mother and child [1]

Maternity care in the Netherlands is known for its high percentage of home births and for the independent situation of midwives [3]. In the Netherlands about 175,000 births occur annually [2]. Obstetric care in the Netherlands is delivered by primary caregivers: midwives or general practitioners (GPs), and by specialist obstetricians. Women with low-risk pregnancies obtain antenatal care from midwives. When they remain low risk throughout pregnancy they are free to choose to give birth either at home or in a hospital. Their midwife support them in their choice and attend the birth in the chosen place. Referral to specialist care only occurs in case of complications [1]

We need to get a clear picture of maternity care and their providers in developed countries though it is assumed that developed countries have better maternal care. There has been ample literature on midwives and maternal care in low resource environments for example see [7]. Furthermore, Human-Computer Interaction (HCI) community is working with midwives to design better systems such as improved ultrasound [5]. To better comprehend and support the workflows of midwives we conducted an interview study to discover the needs of midwives and pregnant women to develop technologies that will help them to better serve their needs.

\section{Understanding the current practice}

In total 15 midwives and 10 pregnant women who were first time pregnant participated in a semistructured interview. They were contacted via the Roya Dutch Organization of Midwives (KNOV) [2] and from local midwifery centers. The participation was voluntary. Each interview session was one hour long. Interview data were audio recorded and later transcribed. The data were thematically analysed by the first author and one external researcher and the qualitative data analysis method described in [6] was followed. The key themes that emerged from the interviews are explained below:

Insights gathered from the midwives

It would be beneficial to have an integrated software system for doctors, midwives and other caregivers. The current system in the Netherlands does not allow different stakeholders to access the same patient data from their own software terminals. In the Netherlands there are four different software systems that are used in midwifery centers to provide antenatal care. Therefore, creating an integrated health care system is a crucial need where privacy of the end user data will be preserved. The use of a particular software is based on price, and recommendations from other colleagues and the associated midwifery centers who work together. Improving the usability and features of the current midwifery software systems used in the midwifery centers will certainly help the workflow of the midwives.

We also found that midwives find their task harder due to attitude of the people which is hard to control. However, it would be helpful if midwife could be virtually connected with the patients which could reduce their workload. Most of the time midwives are overloaded with so many queries of the women who are first time 


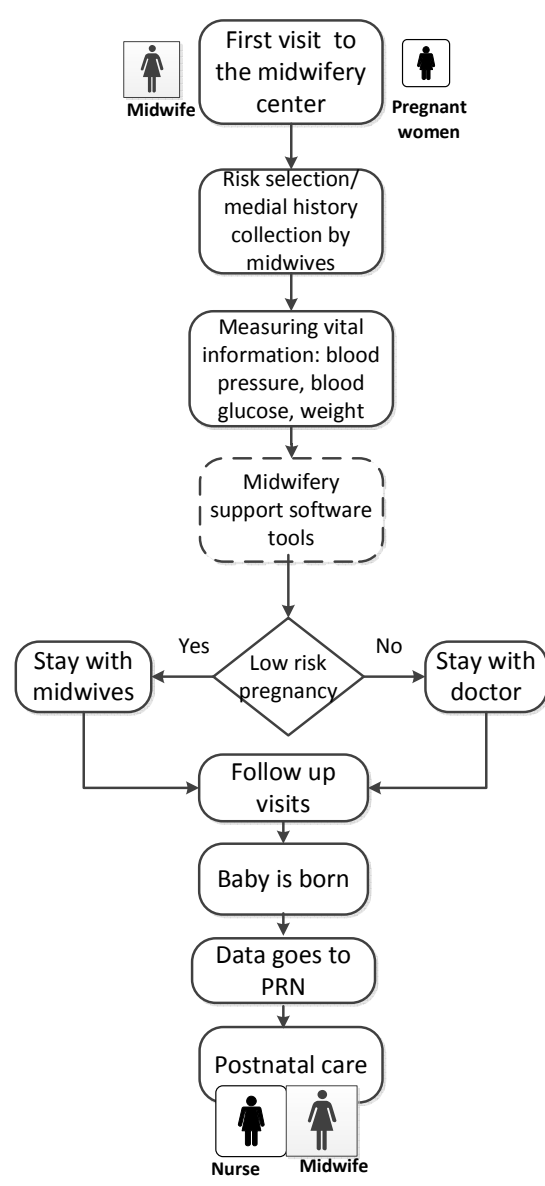

Figure 1. Typical workflow of the Dutch midwifery system pregnant. Therefore, creating a virtual community and getting regular reminders about appointments could help to bridge the situation. One of the issues midwives mentioned that they have problems in postnatal care support as well. The main reason was that the nurses involved in postnatal care are not sure about different measurements such as high bilirubin of the new born child. Most of the time the nurses call the midwives or doctors to consult. We summarize the following insights from the interviews with midwives:

- Improving interoperability and centralization of software for midwives.

- Improving usability and features of the software for midwives for example tools that may provide support while midwives go out of their practice to provide care.

- Creating virtual midwives to reduce workload for midwives and provide support to pregnant women.

- Providing tools to support postnatal care.

Insights gathered from the pregnant women It has also been observed that some pregnant women (7 out of 10) are very motivated and eager to know the growth of their expected baby. Since the midwives check the growth once in a month and sometimes it is hard to follow some appointments it would be beneficial to have a software system that will inform pregnant women about the growth of their baby. Furthermore, the application will help pregnant women to get a piece of mind. In addition this will lower the burden from the midwives for example they will receive less worrying question from the pregnant women in a less frequent manner. Midwives also provide information to pregnant women about their practices and give lot of information about pregnancy such as foods, exercises and follow up visits. Therefore, it is important that the provided in- formation given to pregnant women are easy to remember and manage during the 9 months. We summarized the insights gathered from the pregnant women:

- An application that may help to monitor pregnancy and the growth of the baby over nine months.

- Support for information need that may arise between visits to the midwifery centers.

- Providing guidance to pregnant women that may help pregnant women to take decision regarding do's and don'ts during pregnancy.

\section{Design Explorations}

The findings are encouraging though the prenatal care in the Netherlands is considered very good and some of the findings are due to the nature of the Dutch

healthcare system. The design requirements from the interviews were reviewed in a brainstorming session.

We have adopted to follow participatory design method with local midwives and pregnant women to design improved services for them. We realized that the gathered requirements cannot be implemented in a single system. Therefore, we priorities the design trend in two directions. Firstly, a mobile application which could help to integrate the local midwifery software systems would be helpful for the midwives. We recommend having a mobile app that would help to access the software systems while midwives have to visit a patient. Such kinds of apps are not available to midwives. The mobile app should be able to synchronise the gathered data later on with their own software terminals at the midwifery centers. In order to provide improved care by midwives we also plan to provide virtual midwifery support to pregnant women. For example, we are designing an app for pregnant women. The objective is to help pregnant mothers to get useful information and make them aware about the growth of their babies over 9 months. 
The app will also be able to document each visit to the midwifery centres and will provide recommendations on food and nutrition, exercise etc. Consequently, midwives will be more connected to the pregnant women and virtual support will be provided as well. Furthermore, pregnant women will also find a peace of mind during the whole period of the pregnancy.

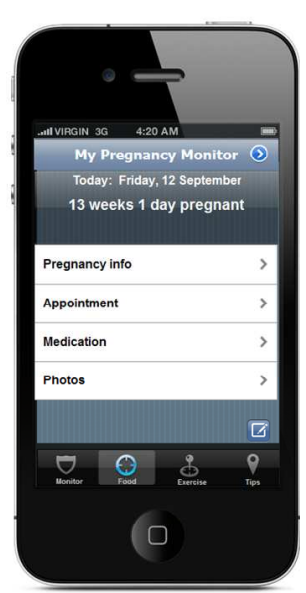

Mobile app for pregnant women

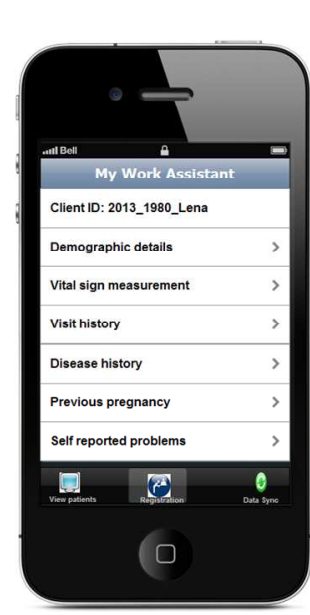

Mobile app for midwives \& nurses

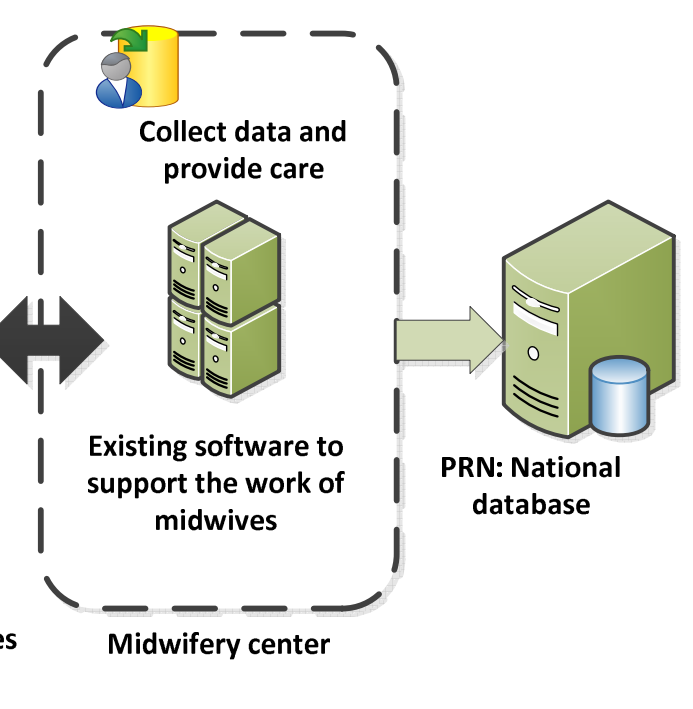

Figure 2. Conceptual model of the proposed system. Pregnant women will use the app to get assistance about their pregnancy related questions such as healthy behaviors, foods and exercises during each trimester. Midwives will use the mobile app when they need to visit a patient. The app for midwife is connected to the local software used in the midwifery center to provide antenatal care. Consequently midwifes are expected to have lower burden during their midwifery practices since the app for pregnant women will also act as a virtual midwifery support. The nurses may use the app during post natal care such as to determine the bilirubin level of the new born babies.

\section{Conclusions and Future Work}

In this paper we presented the preliminary assessment and design of an application to improve the workflow of Dutch midwives. We are working to build the working prototype of the proposed system. We intend to conduct a long term user test to understand how midwives and pregnant women use the application.

\section{Acknowledgements}

We thank all the participants for their cooperation.

\section{Citations}

[1] Bullough, C., N. Meda, et al. (2005). Review: Current strategies for the reduction of maternal mortality. BJOG: An International Journal of Obstetrics \& Gynaecology 112(9) 1180-1188.

[2] Dutch midwifery association. www.knov.nl

[3] Bais, J. M. J. and Pel, M.(2006). The basis of the Dutch obstetric system: risk selection. European Clinics in Obstetrics and Gynaecology 2(4) 209-212.

[4] Herz, B. K. and Measham, A. R. (1987). The safe motherhood initiative: Proposals for ac-tion, World Bank.

[5] Havukumpu, J., Vähäkangas,P., Grönroos, E., and Häkkinen, J. (2006). Midwives experiences of using HMD in ultrasound scan. In Proceedings of the 4th Nordic conference on Human-computer interaction: changing roles (NordiCHI '06), ACM 369-372.

[6] Strauss A., Corbin J.M. (1990). Basics of qualitative research: grounded theory procedures and techniques. Sage Publications, 1990.

[7] Smeenk, A. D. J. and H. A. M. J. ten Have. (2003). Medicalization and obstetric care: an analysis of developments in Dutch midwifery. Medicine, health care and philosophy 6(2) 153-165. 